\title{
Dietary Sodium Restriction in the Management of Chronic Kidney Disease: A Meta-Analysis of RCTs
}

\author{
Sai Sidharth $\mathbf{M}^{1 *}$ and Dhar $\mathbf{M}^{2}$ \\ ${ }^{1}$ Associate Data Scientist, Centre for Open Data Research \\ (CODR) - Public Affairs Centre (PAC), India \\ ${ }^{2}$ Professor, Department of Bio-Statistics and \\ Epidemiology, International Institute for Population \\ Sciences (IIPS), India \\ *Corresponding author: Sai Sidharth M, Associate \\ Data Scientist, Centre for Open Data Research (CODR) - \\ Public Affairs Centre (PAC), Bengaluru, India
}

Received: September 06, 2021; Accepted: October 08, 2021; Published: October 15, 2021

\begin{abstract}
Introduction: Non-pharmacological strategies such as lowering sodium intake aim to protect renal function and delay the initiation of renal replacement therapy. It might also be a cost-effective method to improve Chronic Kidney Disease (CKD) prognosis. We decided to perform a meta-analysis of Randomized Controlled Trials (RCTs) to evaluate the effects of low versus high sodium intake in adults with CKD.
\end{abstract}

Methodology: We searched the online databases - PUBMED, Cochrane Kidney and Transplant Specialized Register, Cochrane Library and Google Scholar to $31^{\text {st }}$ December 2020 for RCTs to be included in the study. MetaAnalysis was performed for the intervention groups for each arm against the control. Inverse variance methods were applied for analysis using random effects models due to the high heterogeneity among the studies.

Results: Our search strategy yielded seven studies from six countries with 465 participants. The overall effect on restricted sodium intake favored reduction in systolic blood pressure with an overall mean difference of $-6.14(95 \% \mathrm{Cl}:-9.52$, -2.76) and reduction in diastolic blood pressure with a mean difference of -3.08 $(95 \% \mathrm{Cl}:-4.62,-1.55)$. There was lowering of estimated Glomerular Filtration Rate (eGFR), however the same was not statistically significant.

Conclusion: The study found that restricted salt intake could significantly reduce systolic and diastolic BP. Further, multi-center RCTs for longer durations across different stages of CKD could effectively assess the effects of restricted sodium intake on vital parameters. Such study designs could also help clinicians identify the optimal intake of dietary sodium to achieve better renal and cardio vascular outcomes.

Keywords: Chronic kidney disease; Meta-analysis; Sodium restricted diet

\section{Introduction}

Non-Communicable Diseases (NCDs) have replaced communicable diseases and is the most common form of morbidity and eventually mortality across the globe $[1,2]$. Among the NCDs, the burden of CKD is on a rise due to the multitude of risk factors associated with the disease. Evidence from literature suggests that CKD affects about $10-16 \%$ of the adults across the globe [3]. The burden of CKD is well established in developed countries but recent studies suggest that CKD burden may be even higher in developing countries [4]. This increase may be related to the increase in rates of hypertension and diabetes [3]. Much of the morbidity and mortality associated with diabetes and hypertension are attributable to kidney disease and its complications. The demographic transition driving this rise is expected to occur predominantly in developing countries and will further continue to challenge the economic capacity of these countries to provide RRT to the already increasing number of people with ESKD [5].

The global action plan for the prevention and control of NCDs (2013-20) outlined an approach to reduce the mortality from four major categories of NCDs namely - cancer, cardiovascular disease, chronic respiratory disease and diabetes by $25 \%$ by the year 2025 [6].
The Global Burden Of Disease (GBD) 2015 study estimated that 1.2 million deaths, 19 million Disability-Adjusted Life Years (DALYs) and 18 million years of life lost from CVDs were directly attributed to reduced GFR [7,8]. It also estimated that in 2015, 1.2 million people died from renal failure, an increase of $32 \%$ since 2005 [8]. In 2010, an estimated 2.3-7.1 million people with ESKD died without access to RRT [9]. It is also possible that limited availability of epidemiological data and poor awareness about the consequences of CKD might seriously underestimate the burden of CKD. This might be a severe complication in regions with inadequate public health systems.

Initiation of RRT or renal transplants for people with ESKD possess serious economic burden. High-income countries typically spend 2-3\% of their health-care budget on the treatment of ESKD even though less than $0.3 \%$ of the total population receive these treatments [10]. In 2010, 2.62 million people received dialysis therapy worldwide and this number is expected to double by 2030 [9]. However, if risk factors related to renal function is identified early, deterioration of renal function can be slowed down by the means of cost-effective interventions, several of which are available on the WHO's 'Best Buys and other recommended interventions for the prevention and control of NCDs' [6] which also includes reduced dietary sodium intake. 
The relation between excess sodium intake with hypertension and kidney disease is well known. In the general population high sodium intake is associated with hypertension and cardio vascular events. It increases the extent of release of albuminuria in CKD patients as well as the general population [11]. RCTs conducted in the past have also shown that reducing sodium intake has significantly reduced systolic $\mathrm{BP}$ and diastolic BP [12]. This cost-effective intervention might help renal impaired patients to delay the initiation of RRT. It is imperative that we perform a systematic review of the available literature to understand the effect of the most commonly recommended dietary intervention in the management of CKD - sodium restricted diet.

\section{Methods}

\section{Search strategy}

The present review was conducted in accordance with the Preferred Reporting Items for Systematic Reviews and Meta-Analysis (PRISMA) guidelines [13]. We searched the online databases PUBMED, Cochrane Kidney and Transplant Specialized Register, Cochrane Library and Google Scholar to 31 December 2020 for RCTs to be included in this meta-analysis. The search strategy was limited to those articles published in English. The following keywords were used as part of the search strategy - chronic kidney disease, CKD, sodium diet, renal disease, renal insufficiency, chronic renal insufficiency, nephropathy, glomerular disease and glomerulonephritis. References of these studies were also screened to further identify potentially relevant studies for the review. The search was carried out from 15th May 2021 till 15th June 2021. Boolean operators such as "OR", "AND" were used in the search strategy. The protocol was registered with PROSPERO International prospective register of systematic reviews vide registration number CRD42021254921.

\section{Inclusion criteria}

We included all RCTs which evaluated the effects of low versus high sodium intake in people with CKD including those with ESKD. The studies included in the meta-analysis should have been published in a peer-reviewed journal. The titles and abstracts of articles from the search strategy were screened and those papers were carefully reviewed before including it in the review using the pre-defined inclusion criteria.

\section{Exclusion criteria}

We excluded studies on pregnant women, on children less than 18 years of age, which were followed up for less than 4 weeks, which did not report data on numbers in exposed and un-exposed categories and number of outcomes in the two categories and when it was impossible to extract data from the published results of the study. The articles which were found in languages other than English were also excluded from the meta-analysis.

\section{Interventions (Exposure)}

We considered the intervention of restricted sodium diet on patients with CKD including those with ESKD. Accordingly, we included studies which were RCTs measuring the effects of low versus high sodium intake in people with CKD and ESKD. The studies included in the review should compare two or more levels of differing sodium intake and should be followed up for at least 4 weeks.

\section{Outcome measurements}

- Change in systolic and diastolic BP
- $\quad$ Change in serum creatinine

- $\quad$ Change in eGFR

- $\quad$ Change in serum urea

\section{Data collection, extraction and analysis}

The above mentioned search strategy was used to obtain titles and abstracts of the studies that could potentially be used in our review. The titles and abstracts were screened and those studies that were deemed to be unsuitable for our review were discarded based on the exclusion criteria. Data from the included studies in the review was extracted using the standard extraction forms available from the Cochrane Library. Only those studies which reported relevant outcome measurements were included in the review. In the studies which reported the outcome measures at different time periods, the most recent one was used for the analysis. We then coded and categorized the interventions used in each of the studies selected for the review. Studies with multiple interventions were analyzed for each arm against control and the analysis was performed separately for each arm. Review Manager (RevMan) version 5.3, The Cochrane Collaboration [14] was used to abstract and collect information about the study characteristics, descriptions of the interventions and comparisons, outcome of interest and effects. We assessed quality based on the CHERG adaptation of the GRADE checklist at individual study level [15].

\section{Assessment of heterogeneity}

Heterogeneity of the studies considered was assessed using a ChiSquared test at $5 \%$ level of significance. In addition, $\mathrm{I}^{2}$ test was also applied. Its values of $25 \%, 50 \%$ and $75 \%$ correspond to low, medium and high levels of heterogeneity respectively [16].

\section{Measurement of treatment effect}

Inverse variance methods were applied for analysis using random effects models. Continuous scales of outcome measurement were used to assess the effects of the interventions. The outcome measurements in the study were continuous (such as serum creatinine, eGFR, albuminuria, proteinuria, Protein Creatinine Ratio (PCR), Albumin Creatinine Ratio (ACR), blood pressure). In such cases, the Mean Difference (MD) is measured if same scales of measurements were used or the Standardized Mean Difference (SMD) if different scales of measurements were used corresponding to 95\% Confidence Interval. In studies reporting measures at different intervals, the scores at the endpoint was used in the meta-analysis. Forest plots were plotted to measure treatment effects and to make inferences.

\section{Results}

\section{Systematic review}

The search strategy yielded 854 results across PUBMED, Cochrane Kidney and Transplant Specialized Register and Google Scholar. After initial screening for title and abstracts 164 records were screened for including in the review. These full-text articles were assessed for inclusion for qualitative synthesis. Further, 157 records were excluded because of Non-RCTs, text not in English and wrong intervention. The remaining 7 studies ( 8 reports) with 465 participants were enrolled in the low-sodium versus standard diet which was used for performing meta-analysis. Figure 1 presents the flow chart of the study selection process for the meta-analysis. 


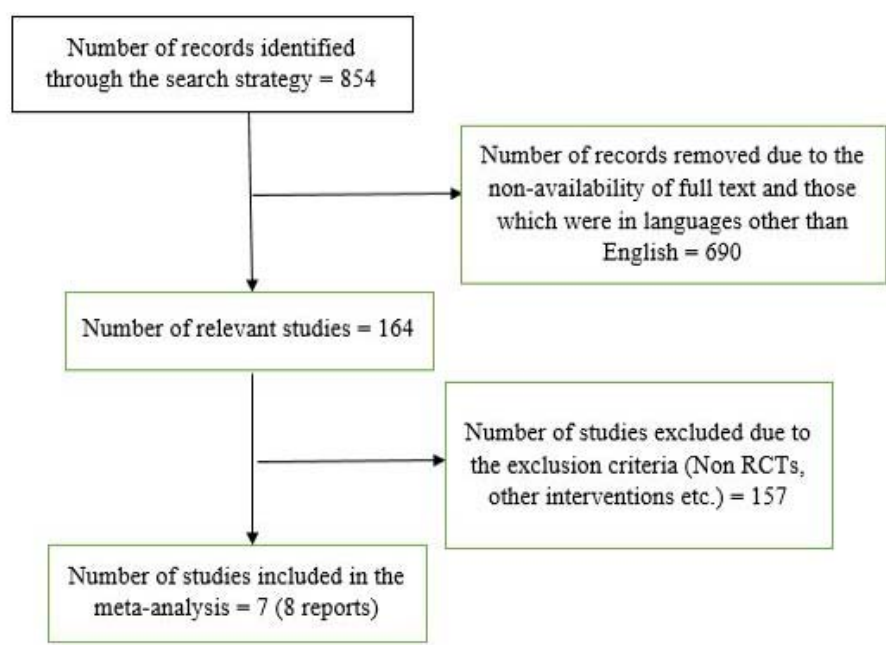

Figure 1: Flow chart of study selection process for dietary sodium restriction in the management of Chronic Kidney Disease (CKD).

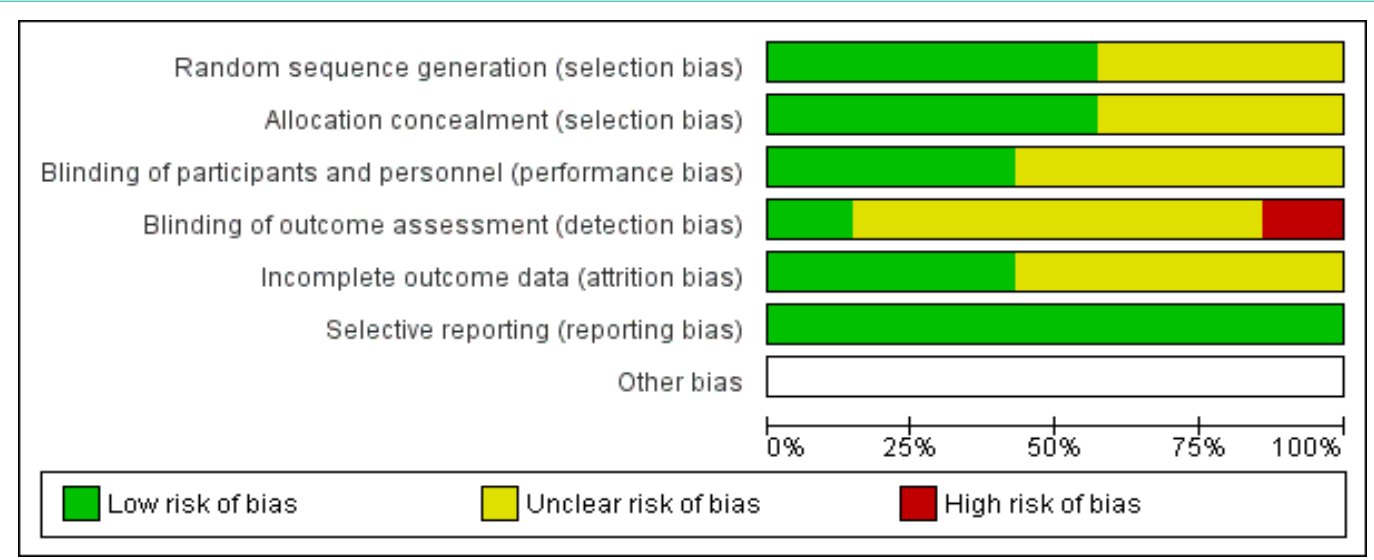

Figure 2: Risk of Bias Summary Graph: Review authors' judgements about each risk of bias item presented as percentages across all studies. Studies were frequently assessed as having low, unclear or high risk of bias for the risk of bias study domains with selection bias, performance bias, attrition bias, reporting bias and other bias.

\section{Risk of bias assessment}

The risk of bias summary graph and the risk of bias summary table are the two commonly used methods to perform evidence synthesis from the studies included in this review [16]. Signaling questions like

- Was there sufficient random sequence generation

- Was there sufficient allocation concealment

- Was there blinding of participants and personnel during the study period

- Was there blinding of outcome assessment

- Was there any selective reporting among the study participants

Were answered based upon the published protocol of the study corresponding to low, unclear and high risk of bias. If the signaling question received an answer of yes, the risk of bias for that study will be low, if the signaling question received an answer of no, then the risk of bias for that study will be high and in instances where the answers to the signaling question might be probably yes or probably no then the risk of bias for that study was considered as unclear risk of bias. Figure 2 summarizes the risk of bias for the included studies.

\section{Random sequence generation}

All included studies in this meta-analysis had used some sort of randomization technique. However, only 4 of them had provided the exact method of randomization [18,19,21,22].

\section{Blinding of participants and personnel}

Only 3 out of the 7 studies had blinded their participants [17-19]. The other studies included in the meta-analysis were either crossover study designs or had not reported the allocation concealment clearly. Also, all the studies had mentioned whether their outcome measurements were blinded or not. One study [18] had asked the study participants to record their BP measurements which is why it corresponds to high risk of bias.

\section{Incomplete outcome data}

Attrition rates were clearly explained in 3 studies [19,21,22]. The rest of the studies did not discuss the reasons for the losses due to follow-up. 


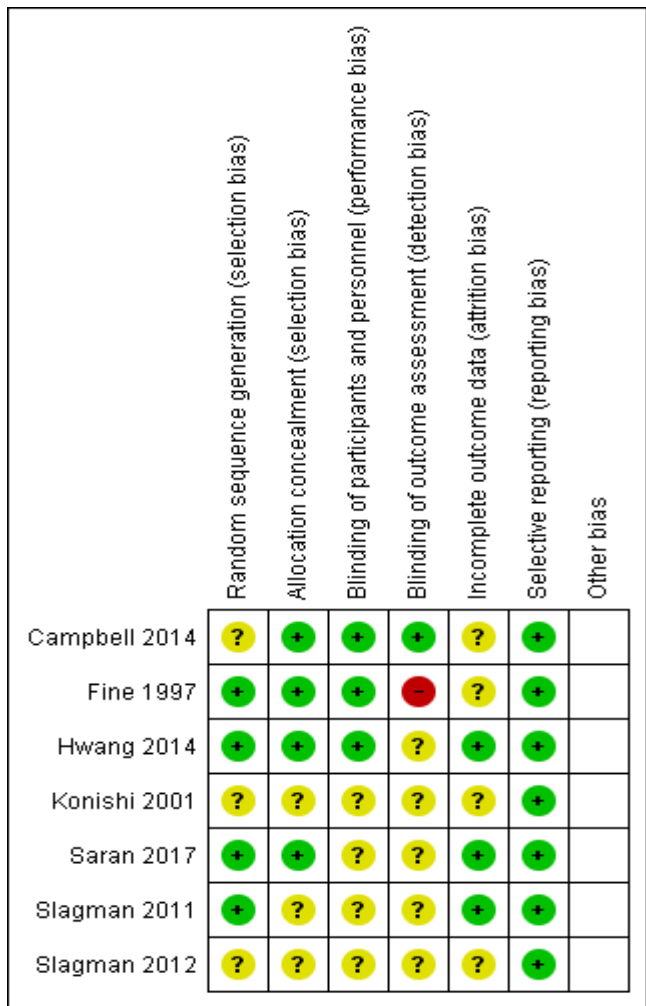

Figure 3: Risk of Bias Summary: Review authors' judgements about each risk of bias item for each included study.

\section{Selective reporting}

All the included studies clearly reported the outcome measurements across both the intervention and control groups. Figure 3 summarizes the risk of bias of each item for the included studies in the review

\section{Pooled effects on different outcomes}

Systolic blood pressure: Six studies [17-21,23] evaluated the systolic BP measurement comparing low and high sodium intake. Figure 4 presents the Forest Plot for the effect of restricted sodium intake on systolic BP among CKD patients across different intervention groups. High heterogeneity was seen among the studies and were statistically significant with $\mathrm{I}^{2}=88 \%$. Therefore, the random effects model was used to summarize the effects reported in different studies. The overall effect favored the restricted sodium intake group with an overall mean difference of -6.14(95\% CI: -9.52, -2.76).

\section{Diastolic blood pressure}

Six studies [17-21,23] evaluated the diastolic BP measurement comparing low and high sodium intake. Figure 5 presents the Forest Plot for the effect of restricted sodium intake on diastolic BP among CKD patients across different intervention groups. Random effects model was used to summarize the effects reported in these studies due to high heterogeneity among the studies. The overall effect favored the experimental group with a mean difference of -3.08 (95\% CI: -4.62 , $-1.55)$. Heterogeneity among the studies was statistically significant with $\mathrm{I}^{2}=79 \%$.

\section{Serum creatinine}

Four studies [17,19,21,22] evaluated the effects of sodium restriction on serum creatinine. Studies which reported median and inter quartile range were converted to mean and standard deviation using the R package estmeansd() [24]. Figure 6 presents the effects of restricted sodium diet among CKD patients on serum creatinine across different groups. In the sodium restricted dietary group, the overall effect favored an increase in serum creatinine with a slight increase in mean difference of $0.17(95 \%$ CI: $0.09,0.26)$ and the heterogeneity among the studies was statistically significant with $I^{2}=76 \%$.

\section{Estimated glomerular filtration rate}

Three studies $[17,19,21]$ evaluated the effects of restricted sodium diet with the control on eGFR. Figure 7 presents the effects of restricted sodium diet on eGFR across different intervention groups. In the sodium restricted dietary group there was decrease in mean difference of -3.59 (95\% CI: -7.91, 0.74) and the heterogeneity statistic $\mathrm{I}^{2}=90 \%$ was statistically significant.

\section{Serum urea}

One study [22] evaluated the effect of restricted sodium intake on serum urea. Figure 8 presents the forest plot for the effect of restricted sodium intake on serum urea across each intervention group. Random effects model was used to summarize the effects reported in these studies. In the study which was included for meta-analysis, the overall effect favored an increase in serum urea and provided statistically significant results with $\mathrm{I}^{2}=91 \%$ and the mean difference being 6.58(95\% CI: 4.66, 8.50).

\begin{tabular}{|c|c|c|c|c|c|c|c|c|c|c|}
\hline \multirow{2}{*}{$\begin{array}{l}\text { Study or Subgroup } \\
\text { Campbell } 2014\end{array}$} & \multicolumn{3}{|c|}{ Experimental } & \multicolumn{3}{|c|}{ Control } & Weight & $\begin{array}{l}\text { Mean Difference } \\
\text { IV, Random, } 95 \% \mathrm{Cl}\end{array}$ & \multicolumn{2}{|c|}{$\begin{array}{c}\text { Mean Difference } \\
\text { IV, Random, 95\% Cl }\end{array}$} \\
\hline & 144.9 & 13.1 & 20 & 154.6 & 11.9 & 20 & $10.9 \%$ & $-9.70[-17.46,-1.94]$ & $=$ & \\
\hline Fine 1997 & 135 & 19 & 20 & 144 & 21 & 20 & $5.8 \%$ & $-9.00[-21.41,3.41]$ & & \\
\hline Hwang 2014 & 121.2 & 1.3 & 119 & 122.6 & 1.3 & 126 & $25.7 \%$ & $-1.40[-1.73,-1.07]$ & 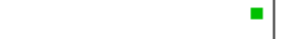 & \\
\hline Konishi 2001 & 118 & 10 & 18 & 127 & 8 & 18 & $14.4 \%$ & $-9.00[-14.92,-3.08]$ & & \\
\hline Saran 2017 & 132.6 & 17.1 & 29 & 143.6 & 18.7 & 27 & $8.6 \%$ & $-11.00[-20.41,-1.59]$ & & \\
\hline Saran 2017 & 134.3 & 18.2 & 29 & 139.1 & 13.8 & 29 & $10.1 \%$ & $-4.80[-13.11,3.51]$ & & \\
\hline Slagman 2012 & 137 & 3 & 32 & 143 & 3 & 32 & $24.6 \%$ & $-6.00[-7.47,-4.53]$ & $\rightarrow$ & \\
\hline Total $(95 \% \mathrm{Cl})$ & & & 267 & & & 272 & $100.0 \%$ & $-6.14[-9.52,-2.76]$ & & \\
\hline \multicolumn{9}{|c|}{$\begin{array}{l}\text { Heterogeneity: } \mathrm{Tau}^{2}=11.54 ; \mathrm{Chi}^{2}=51.65, \mathrm{df}=6(\mathrm{P}=0.00001) ; \mathrm{I}^{2}=88 \% \\
\text { Test for overall effect: } Z=3.56(\mathrm{P}=0.0004)\end{array}$} & $\begin{array}{ccc}-20 & -10 & 0 \\
\text { Favours [experimental] }\end{array}$ & ${ }_{10}^{10} 20$ \\
\hline
\end{tabular}




\begin{tabular}{|c|c|c|c|c|c|c|c|c|c|c|}
\hline \multirow[b]{2}{*}{ Study or Subgroup } & \multicolumn{3}{|c|}{ Experimental } & \multicolumn{3}{|c|}{ Control } & \multirow[b]{2}{*}{ Weight } & \multirow{2}{*}{$\begin{array}{l}\text { Mean Difference } \\
\text { IV, Random, } 95 \% \text { Cl }\end{array}$} & \multirow{2}{*}{\multicolumn{2}{|c|}{$\begin{array}{c}\text { Mean Difference } \\
\text { IV, Random, } 95 \% \mathrm{Cl}\end{array}$}} \\
\hline & Mean & SD & Total & Mean & SD & Total & & & & \\
\hline Campbell 2014 & 79.4 & 9.4 & 20 & 83.3 & 9 & 20 & $6.0 \%$ & $-3.90[-9.60,1.80]$ & & 7 \\
\hline Fine 1997 & 77 & 8 & 20 & 82 & 12 & 20 & $5.0 \%$ & $-5.00[-11.32,1.32]$ & & - \\
\hline Hwang 2014 & 73.6 & 0.9 & 119 & 74.8 & 0.9 & 126 & $34.4 \%$ & $-1.20[-1.43,-0.97]$ & - & \\
\hline Konishi 2001 & 77 & 7 & 18 & 82 & 5 & 18 & $10.4 \%$ & $-5.00[-8.97,-1.03]$ & & \\
\hline Saran 2017 & 71.2 & 8.6 & 29 & 76.8 & 13.4 & 27 & $5.6 \%$ & $-5.60[-11.55,0.35]$ & & \\
\hline Saran 2017 & 71.8 & 11.7 & 29 & 77.7 & 8.7 & 29 & $6.7 \%$ & $-5.90[-11.21,-0.59]$ & & \\
\hline Slagman 2012 & 83 & 1 & 32 & 86 & 2 & 32 & $31.8 \%$ & $-3.00[-3.77,-2.23]$ & - & \\
\hline Total $(95 \% \mathrm{Cl})$ & & & 267 & & & 272 & $100.0 \%$ & $-3.08[-4.62,-1.55]$ & & \\
\hline \multicolumn{9}{|c|}{$\begin{array}{l}\text { Heterogeneity: } \operatorname{Tau}^{2}=1.77 ; \mathrm{Chi}^{2}=29.16, \mathrm{df}=6(\mathrm{P}<0.0001) ;\left.\right|^{2}=79 \% \\
\text { Test for overall effect: } Z=3.94(P<0.0001)\end{array}$} & $\begin{array}{cc}-10 & -5 \\
\text { Favours [experimental] }\end{array}$ & $\begin{array}{lrr}0 & 5 & 10 \\
0 & 10 \\
\text { Favours [control] }\end{array}$ \\
\hline
\end{tabular}

Figure 5: Forest plot comparing the effects of low and high sodium intake on diastolic blood pressure.

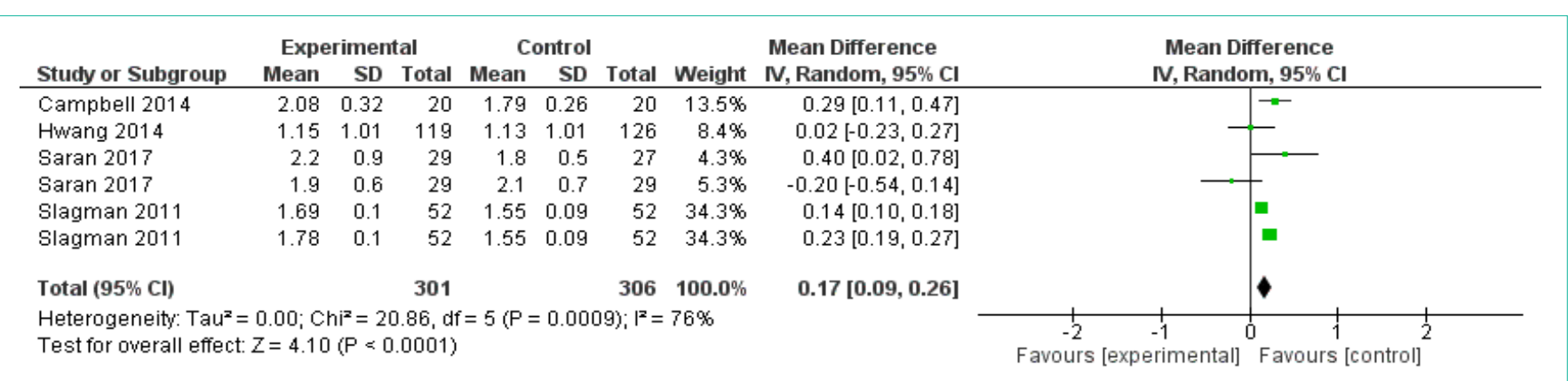

Figure 6: Forest plot comparing the effects of low and high sodium intake on serum creatinine.

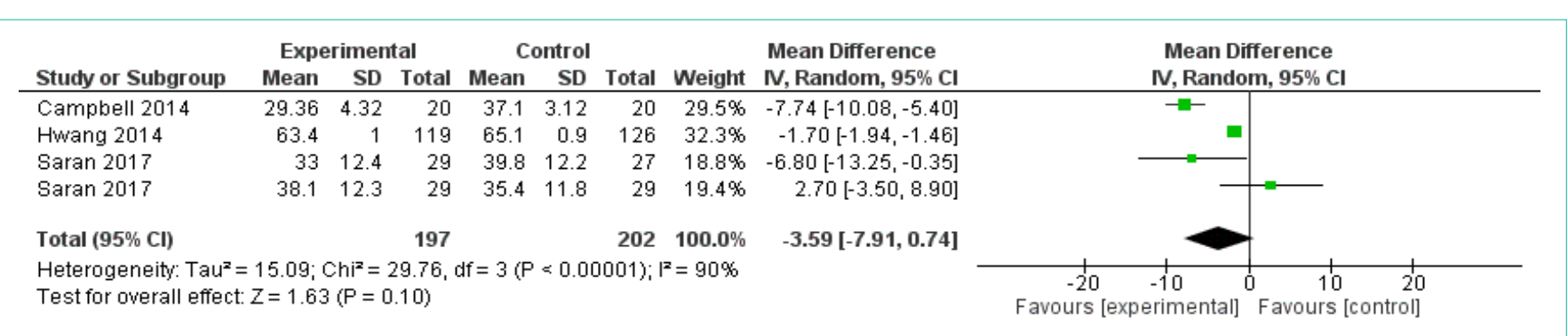

Figure 7: Forest plot comparing the effects of low and high sodium intake on estimated glomerular filtration rate.

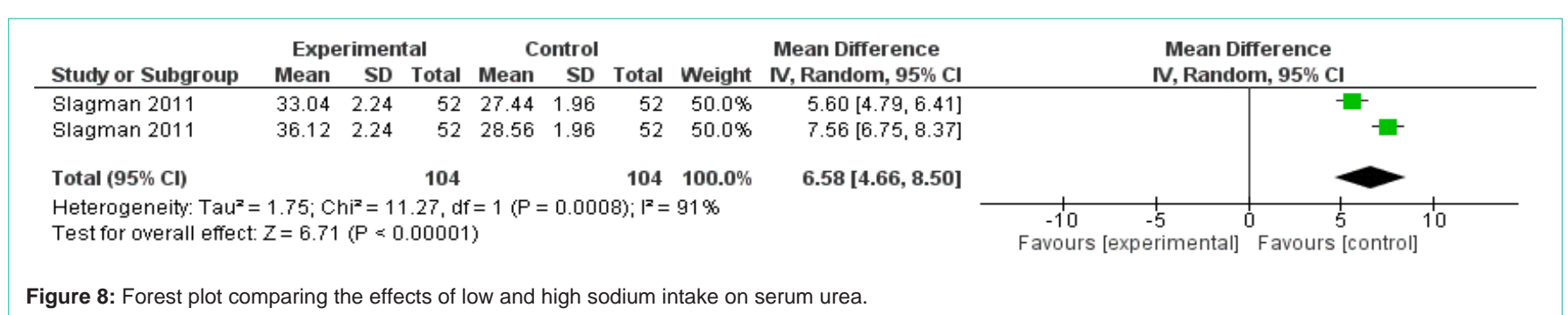

\section{Discussion}

There are millions of people across the globe who are suffering from CKD and many of them have to take up RRT to sustain their life while some die without access to RRT. Actual burden may be far more than reported as due to limited awareness about the severity of the disease and lack of epidemiological data there is a serious possibility of underestimation of the burden of CKD. This might have severe repercussions in future especially in regions with inadequate health systems. People with ESKD are at higher risk of mortality in comparison to the general population. The damage to their kidneys are irreversible and management strategies of CKD are aimed at treatment of existing conditions like controlling BP, diabetes and decreasing albuminuria. Important treatment goals for CKD management includes reducing the risk of heart disease by BP management and reduction of albuminuria. Costs of treating people with ESKD have been estimated to be about 10 times more than that of CKD management [2]. Nutritional strategies like modifying sodium intake may help in preserving renal function and defer the initiation of RRT. There are many studies conducted to assess the effects of low 
sodium diets on BP and renal parameters [25-27]. However, there is large heterogeneity in the effects of the interventions reported by different studies. Moreover, from a broader perspective, one needs to find the overall effect of interventions under consideration on different outcomes. Thus, we decided to perform a meta-analysis of RCTs to test the hypothesis that dietary sodium restriction reduces BP and renal parameters and also to quantify overall effects in terms of pooled mean difference. We were able to assess the effects of sodium dietary restrictions on BP and renal parameters such as serum creatinine, serum urea and eGFR. However, albuminuria could not be meta-analyzed.

Our study indicated that sodium restriction promises to be a modifiable risk factor for reducing cardiovascular risk and ESKD progression. Earlier meta-analyses [25-27] have all showed that reduction in dietary sodium has reduced $\mathrm{BP}$ and proteinuria which are the cornerstones of CKD management. Thus, present study has endorsed the findings of earlier studies. High sodium intake is associated with risk factors for both heart disease and can cause severe damage to the kidneys. High sodium intake also has a positive association with high BP, proteinuria and fluid overload. Hence, it becomes doubly important for people with CKD to adopt a low sodium dietary regime due to its role in salt balance [12]. Further, low-sodium diet can also reduce arterial stiffness and left ventricular diastolic dysfunction $[28,29]$.

In the present meta-analysis of RCTs, we tried to find whether sodium restriction is efficacious and if it produces significant improvement in systolic BP, diastolic BP and renal parameters like serum creatinine, serum urea and eGFR. Our systematic search strategy after applying the pre-defined inclusion and exclusion criteria yielded 7 studies (8 reports) which included 465 participants enrolled in restricted sodium intake versus control. The minimum follow-up period for inclusion of studies for meta-analysis was four weeks. The RCTs were conducted in as many as 6 countries namely - Australia, Canada, Korea, Japan, United States of America and Netherlands with majority of them being conducted in renal clinic settings. The risk of bias in the studies included in this review appears to be low and unclear. All the studies included in the review had used some sort of randomization technique with 4 of them providing the exact method of randomization. 3 studies had blinded their participants while the other studies were either crossover study designs or had not reported the allocation concealment clearly. The losses due to followup were mentioned in 3 studies while the rest did not exactly specify the reasons for the losses. Also, all studies which were included in the review had clearly reported the outcome measurements across the intervention and control groups.

This study possessed some limitations that need to be considered along with the conclusions. Main limitation was non-inclusion of the studies demanding money for access. Studies included had a follow-up for short durations and hence primary endpoints such as mortality or CVDs could not be assessed and this might also be a possible reason for the differential effects on serum urea, serum creatinine and eGFR. Among the studies included in the review, there was limited evidence to study related outcome measurements such as albuminuria, serum uric acid, serum potassium etc. We were also not able to study the effects of intervention across different stages of CKD due to the insufficient sample size. To overcome these limitations, a major study with enough budgetary provisions may be conducted.

\section{Conclusion}

In conclusion, our study found that restricted salt intake could significantly reduce systolic BP and diastolic BP. This low-cost intervention would be a really good strategy to reduce BP and defer the initiation of RRT. Further, multi-center RCTs for longer durations across different stages of CKD could effectively assess the effects of restricted sodium intake on vital renal and cardio vascular parameters. Such study designs could also help clinicians identify the optimal intake of dietary sodium to achieve better renal and cardio vascular outcomes. Therefore, such studies are recommended.

\section{References}

1. World Health Organization, Public Health Agency of Canada, \& Canada. Public Health Agency of Canada. Preventing chronic diseases: a vital investment. World Health Organization. 2005.

2. Zhang QL, Rothenbacher D. Prevalence of chronic kidney disease in population-based studies: systematic review. BMC public health. 2008; 8: 1-13.

3. Coresh J, Selvin E, Stevens LA, Manzi J, Kusek JW, Eggers P. Prevalence of chronic kidney disease in the United States. 2007; 298: 2038-2047.

4. Luyckx VA, Tuttle KR, Garcia-Garcia G, Gharbi MB, Heerspink HJ, Johnson DW. Reducing major risk factors for chronic kidney disease. Kidney international supplements. 2017; 7: 71-87.

5. Lozano R, Naghavi M, Foreman K, Lim S, Shibuya K, Aboyans V. Global and regional mortality from 235 causes of death for 20 age groups in 1990 and 2010: a systematic analysis for the Global Burden of Disease Study 2010. 2012; 380: 2095-2128.

6. World Health Organization. Global action plan for the prevention and control of noncommunicable diseases 2013-2020. World Health Organization. 2013.

7. Kassebaum NJ, Arora M, Barber RM, Bhutta ZA, Brown J, Carter A. GBD 2015 DALYS and HALE Collaborators. Global, regional, and national disabilityadjusted life-years (DALYs) for 315 diseases and injuries and healthy life expectancy (HALE), 1990-2015: a systematic analysis for the Global Burden of Disease Study 2015. Lancet. 2016; 388: 1603-1658.

8. Wang H, Naghavi M, Allen C, Barber RM, Bhutta ZA, Carter A. GBD 2015 Mortality and Causes of Death Collaborators. Global, regional, and national life expectancy, all-cause mortality, and cause-specific mortality for 249 causes of death, 1980-2015: a systematic analysis for the Global Burden of Disease Study 2015. Lancet. 2016; 388: 1459-1544.

9. Liyanage T, Ninomiya T, Jha V, Neal B, Patrice HM, Okpechi I. Worldwide access to treatment for end-stage kidney disease: a systematic review. Lancet. 2015; 385: 1975-1982.

10. Couser WG, Remuzzi G, Mendis S and Tonelli M. The contribution of chronic kidney disease to the global burden of major noncommunicable diseases. 2011; 80: 1258-1270.

11. Ritz E, Koleganova $\mathrm{N}$ and Piecha $\mathrm{G}$. Role of sodium intake in the progression of chronic kidney disease. Journal of Renal Nutrition. 2009; 19: 61-62.

12. McMahon EJ, Bauer JD, Hawley CM, Isbel NM, Stowasser M, Johnson DW. A randomized trial of dietary sodium restriction in CKD. Journal of the American Society of Nephrology. 2013; 24: 2096-2103.

13. Moher D, Liberati A, Tetzlaff J, Altman DG. PRISMA Group. Preferred reporting items for systematic reviews and meta-analyses: the PRISMA statement. 2009; 6: e1000097.

14. Review Manager (RevMan) [Computer program]. Version 5.3. Copenhagen: The Nordic Cochrane Centre, The Cochrane Collaboration. 2014.

15. GRADE Working Group. Grading quality of evidence and strength of recommendations. 2004; 19: 328: 1490. 
16. Higgins JP, Thomas J, Chandler J, Cumpston M, Li Tand Page MJ, et al editor(s). Cochrane Handbook for Systematic Reviews of Interventions Version 6.1, Cochrane, 2020. Available from training.cochrane.org/handbook.

17. Campbell KL, Johnson DW, Bauer JD, Hawley CM, Isbel NM, Stowasser M A randomized trial of sodium-restriction on kidney function, fluid volume and adipokines in CKD patients. 2014; 15: 1-6.

18. Fine A, Fontaine B and Ma M. Commonly prescribed salt intake in continuous ambulatory peritoneal dialysis patients is too restrictive: results of a doubleblind crossover study. 1997; 8: 1311-1314.

19. Hwang JH, Chin HJ, Kim S, Kim DK, Kim S, Park JH. Effects of intensive low-salt diet education on albuminuria among nondiabetic patients with hypertension treated with olmesartan: a single-blinded randomized, controlled trial. 2014; 9: 2059-2069.

20. Konishi Y, Okada N, Okamura M, Morikawa T, Okumura M, Yoshioka K. Sodium sensitivity of blood pressure appearing before hypertension and related to histological damage in immunoglobulin a nephropathy. 2001; 38 : 81-85.

21. Saran R, Padilla RL, Gillespie BW, Heung M, Hummel SL, Derebail VK. A randomized crossover trial of dietary sodium restriction in stage 3-4 CKD. 2017; 12: 399-407.

22. Slagman MC, Waanders F, Hemmelder MH, Woittiez AJ, Janssen WM, Heerspink HJL. Moderate dietary sodium restriction added to angiotensin converting enzyme inhibition compared with dual blockade in lowering proteinuria and blood pressure: randomised controlled trial. 2011; 26: 343.
23. Slagman MC, Kwakernaak AJ, Yazdani S, Laverman GD, van den Born J, Titze J. Vascular endothelial growth factor $C$ levels are modulated by dietary salt intake in proteinuric chronic kidney disease patients and in healthy subjects. Nephrology Dialysis Transplantation. 2012; 27: 978-982.

24. Sean McGrath, XiaoFei Zhao, Russell Steele and Andrea Benedetti. Estimating the Sample Mean and Standard Deviation from Commonly Reported Quantiles in Meta-Analysis. 2020.

25. McMahon EJ, Campbell KL, Bauer JD, Mudge DW. Altered dietary salt intake for people with chronic kidney disease. 2015; 2: CD010070.

26. Garofalo C, Borrelli S, Provenzano M, De Stefano T, Vita C, Chiodini $P$. Dietary salt restriction in chronic kidney disease: a meta-analysis of randomized clinical trials. 2018; 10: 732.

27. D'Elia L, Rossi G, di Cola MS, Savino I, Galletti F, Strazzullo P. Meta-analysis of the effect of dietary sodium restriction with or without concomitant reninangiotensin-aldosterone system-inhibiting treatment on albuminuria. 2015; 10: 1542-1552.

28. Oberleithner $H$, Riethmüller $C$, Schillers $H$, MacGregor GA, de Wardener Hugh E. et al. Plasma sodium stiffens vascular endothelium and reduces nitric oxide release. 2007, 104, 16281-16286.

29. Al-Solaiman Y, Jesri A, Zhao Y, Morrow JD, Egan BM. Low-Sodium DASH reduces oxidative stress and improves vascular function in salt-sensitive humans. 2009; 23: 826-835. 